\title{
ON INEQUALITIES FOR INTEGRAL OPERATORS
}

\author{
by G. O. OKIKIOLU
}

(Received 7 March, 1969; revised 1 July, 1969)

1. Introduction. In two papers [3] and [4], the author has extended the inequality of Schur (Theorem 319 of [2]) to cases involving kernels which satisfy identities of the form

$$
|\psi(a x, a t)|=|a|^{\nu-1}|\psi(x, t)| \text {. }
$$

The purpose of this paper is to prove a general inequality, which includes the above and also the inequality of Young (Theorem 281 of [2]) as special cases. We shall give the results a general setting by considering functions defined on abstract measure spaces. From this we shall deduce an extension to $n$ dimensions of the results given in [3], which also generalises a similar extension of the Schur inequality given by Stein and Weiss. In fact some cases of the other results given in [5] will follow directly from our theorem.

I am grateful to the referee for his helpful suggestions and comments, which have considerably improved the paper.

We shall consider two measure spaces $\left(S_{1}, A_{1}, \sigma_{1}\right)$ and $\left(S_{2}, A_{2}, \sigma_{2}\right)$ respectively, and denote by $L^{p} \equiv L^{p}(S)$ the space of complex-valued measurable functions on some measure space $(S, A, \sigma)$ such that

$$
\|f\|_{p}=\left(\int_{S}|f|^{p} d \sigma\right)^{1 / p}<\infty .
$$

The numbers $p$ and $p^{\prime}$ will be connected by $1 / p+1 / p^{\prime}=1$.

The Euclidean $n$-space will be denoted by $E_{n}$, so that $x \in E_{n}$ implies $x=\left(x_{1}, x_{2}, \ldots, x_{n}\right)$, where $x_{1}, x_{2}, \ldots$ are real numbers. The norm $|\cdot|$ in $E_{n}$ is then given by

$$
|x|=\left(x_{1}^{2}+x_{1}^{2}+\ldots+x_{n}^{2}\right)^{ \pm} \text {. }
$$

2. The general inequality.

THEOREM 2.1. Let $p, r, \mu_{1}, \mu_{2}$ be positive numbers such that $1<p \leqq r$,

$$
\mu_{1} / p^{\prime}+\mu_{2} / r=1
$$

Let also $\psi$ be a complex-valued function measurable on $S_{2} \times S_{1}$, and suppose that there exist measurable functions $\phi_{1}: S_{1} \rightarrow(0,+\infty), \phi_{2}: S_{2} \rightarrow(0,+\infty)$, and non-negative constants $M_{1}$, $M_{2}$ such that

and

$$
\left.\int_{S_{1}}\left(\phi_{1}(t)\right)^{p^{\prime}}|\psi(x, t)|^{\mu_{1}} d \sigma_{1}(t) \leqq M_{1}^{p^{\prime}}\left(\phi_{2}(x)\right)^{p^{\prime}} \quad \text { (a.e. on } S_{2}\right)
$$

$$
\left.\int_{S_{2}}\left(\phi_{2}(x)\right)^{r}|\psi(x, t)|^{\mu_{2}} d \sigma_{2}(x) \leqq M_{2}^{r}\left(\phi_{1}(t)\right)^{r} \quad \text { (a.e. on } S_{1}\right)
$$


(i) If $T$ is given by

$$
T(f)(x)=\int_{S_{1}} f(t) \psi(x, t) d \sigma_{1}(t) \quad\left(x \in S_{2}\right),
$$

where $f \in L^{p}\left(S_{1}\right)$, then $T: L^{p}\left(S_{1}\right) \rightarrow L^{r}\left(S_{2}\right)$, and, for each $f \in L^{p}\left(S_{1}\right)$,

(ii) If $T^{*}$ is given by

$$
\|T(f)\|_{r} \leqq M_{1} M_{2}\|f\|_{p} \text {. }
$$

$$
T^{*}(g)(t)=\int_{S_{2}} g(x) \psi(x, t) d \sigma_{2}(x) \quad\left(t \in S_{1}\right),
$$

where $g \in L^{r^{\prime}}\left(S_{2}\right)$, then $T^{*}: L^{r^{\prime}}\left(S_{2}\right) \rightarrow L^{p^{\prime}}\left(S_{1}\right)$ and, for each $g \in L^{r^{\prime}}\left(S_{2}\right)$,

$$
\left\|T^{*}(g)\right\|_{p^{\prime}} \leqq M_{1} M_{2}\|g\|_{r^{\prime}}
$$

(iii) If $f \in L^{p}\left(S_{1}\right)$ and $g \in L^{r^{\prime}}\left(S_{2}\right)$, then

$$
\int_{S_{1}} f(t) T^{*}(g)(t) d \sigma_{1}(t)=\int_{S_{2}} g(x) T(f)(x) d \sigma_{2}(x) .
$$

Proof. Since $1<r^{\prime} \leqq p^{\prime}$ and $r=\left(r^{\prime}\right)^{\prime}$, the result (ii) follows immediately from (i). Further, the identity in (iii) follows directly from (i) and (ii), in virtue of Fubini's theorem. It is therefore sufficient to prove (i).

Suppose first that $r>p$, and let $c=1 / p-1 / r$. Then $c>0$ and $1 / p^{\prime}+1 / r+c=1$, whence, by the three-term Hölder inequality with indices $p^{\prime}, r, 1 / c$,

$$
\begin{aligned}
|T(f)(x)| & \leqq \int_{S_{1}}|f||\psi| d \sigma_{1} \\
& =\int_{S_{1}}\left(\phi_{1}|\psi|^{\mu_{1} / p^{\prime}}\right)\left(\phi_{1}^{-1}|\psi|^{\mu_{2} / r}|f|^{p / r}\right)|f|^{p c} d \sigma_{1} \\
& \leqq\left(\int_{S_{1}} \phi_{1}^{p^{\prime}}|\psi|^{\mu_{1}} d \sigma_{1}\right)^{1 / p}\left(\int_{S_{1}} \phi_{1}^{-r}|\psi|^{\mu_{2}}|f|^{p} d \sigma_{1}\right)^{1 / r}\left(\int_{S_{1}}|f|^{p} d \sigma_{1}\right)^{c} \\
& \leqq M_{1}\|f\|_{p}^{p c} \phi_{2}\left(\int_{S_{1}} \phi_{1}^{-r}|\psi|^{\mu_{2}}|f|^{p} d \sigma_{1}\right)^{1 / r},
\end{aligned}
$$

a.e. on $S_{2}$. Raising both sides to the $r$ th power, integrating over $S_{2}$, and using Fubini's theorem on the right, we deduce that

as required.

$$
\begin{aligned}
\int_{S_{2}}|T(f)|^{r} d \sigma_{2} & \leqq M_{1}^{r}\|f\|_{p}^{p c r} \int_{S_{1}} \phi_{1}^{-r}|f|^{p} d \sigma_{1} \int_{S_{2}} \phi_{2}^{r}|\psi|^{\mu_{2}} d \sigma_{2} \\
& \leqq M_{1}^{r} M_{2}^{r}\|f\|_{p}^{p c r} \int_{S_{1}}|f|^{p} d \sigma_{1} \\
& =M_{1}^{r} M_{2}^{r}\|f\|_{p}^{r},
\end{aligned}
$$


There remains the case $r=p$, where $c=0$, and that follows by a closely related argument using the two-term Hölder inequality.

Remark. If $\psi$ is a complex-valued function measurable on $S_{2} \times S_{1}$ such that

$$
\left.\int_{S_{2}}|\psi(x, t)|^{r} d \sigma_{2}(x) \leqq M_{2}^{r} \quad \text { (a.e. on } S_{1}\right) \text {, }
$$

where $1 \leqq r<+\infty, M_{2}$ is a non-negative constant, and $T(f)$ is given by 2.1.3., then

$$
T: L^{1}\left(S_{1}\right) \rightarrow L^{r}\left(S_{2}\right)
$$

and, for each $f \in L^{1}\left(S_{1}\right)$,

$$
\|T(f)\|_{r} \leqq M_{2}\|f\|_{1} .
$$

This is a familiar and simple application of Hölder's inequality, which can be interpreted as the case $p=1$ of Theorem 2.1 (i).

3. Special cases. We shall now consider some special cases of Theorem 2.1. To start with we have the following result.

THEOREM 3.1. (Young's Inequality). Let $f \in L^{p}\left(E_{n}\right), g \in L^{q}\left(E_{n}\right), p \geqq 1, q \geqq 1,1 / r=(1 / p)+$ $(1 / q)-1 \geqq 0$, and let

$$
T(f)(x)=\int_{E_{n}} f(t) g(x-t) d t \quad\left(x \in E_{n}\right)
$$

Then

$$
\|T(f)\|_{r} \leqq\|g\|_{q}\|f\|_{p} .
$$

Proof. The conclusion is easily seen to correspond to the case $S_{1}=S_{2}=E_{n}(n \geqq 1)$, $\phi_{1}(t)=\phi_{2}(t)=1, \mu_{1}=\mu_{2}=q, \psi(x, t)=g(x-t), M_{1}=M_{2}=\|g\|_{q}$ of Theorem 2.1.

Next we consider extensions of the results given in [3]. These will be applied in proving some special cases of results involving fractional integrals obtained in [1] and [5].

THEOREM 3.2. Let $p, r, \mu_{1}, \mu_{2}$ be positive numbers such that

$$
1<p \leqq r, \quad \mu_{1} / p^{\prime}+\mu_{2} / r=1,
$$

let $m, n$ be positive integers, and let $K$ be a complex-valued function measurable on $(0, \infty) \times(0, \infty)$ such that

(i) $|K(a u, a v)|=a^{-\left(n / p^{\prime}+m / r\right)}|K(u, v)|(a>0, u>0, v>0)$,

(ii) $\rho_{n} \int_{0}^{+\infty} v^{r p^{\prime}+\left(n \mu_{1} / p^{\prime}\right)-1}|K(1, v)|^{\mu_{1}} d v=M_{1}^{p^{\prime}}<+\infty$,

(iii) $\rho_{m} \int_{0}^{+\infty} u^{\gamma r+\left(m \mu_{2} / r\right)-1}|K(u, 1)|^{\mu_{2}} d u=M_{2}^{r}<+\infty$, 
where $\gamma$ is any given real number, and $\rho_{n}$ is the surface area measure of the unit sphere in $E_{n}$. Then the conclusions (i), (ii), (iii), of Theorem 2.1 hold with $S_{1}=E_{n}, S_{2}=E_{m}$, and $\psi(x, t)=$ $K(|x|,|t|)$.

Proof. Suppose that, for some real number $\lambda, K$ satisfies the condition

$$
|K(a u, a v)|=a^{-\lambda}|K(u, v)|(a>0, u>0, v>0) .
$$

Taking $\phi_{1}(t)=|t|^{\alpha}, \phi_{2}(x)=|x|^{\beta}, \psi(x, t)=K(|x|,|t|)$, we see that the condition (2.1.1) of Theorem 2.1 becomes

$$
\begin{aligned}
M_{1}^{p^{\prime}}|x|^{\beta p^{\prime}} & \geqq \int_{E_{n}}|t|^{\alpha p^{\prime}}|K(|x|,|t|)|^{\mu_{1}} d t \\
& =|x|^{\alpha p^{\prime}+n} \int_{E_{n}}|\xi|^{\alpha p^{\prime}}|K(|x|,|x||\xi|)|^{\mu_{1}} d \xi \\
& =|x|^{\alpha p^{\prime}+n-\lambda \mu_{1}} \int_{E_{n}}|\xi|^{\alpha p^{\prime}}|K(1,|\xi|)|^{\mu_{1}} d \xi
\end{aligned}
$$

and this is obviously satisfied for all $x \in E_{m}$ if we take

and

$$
\beta=\alpha+n / p^{\prime}-\lambda \mu_{1} / p^{\prime}
$$

$$
M_{1}^{p^{\prime}}=\int_{E_{n}}|\xi|^{\alpha p^{\prime}}|K(1,|\xi|)|^{\mu_{1}} d \xi
$$

Similarly the condition 2.1.2 of Theorem 2.1 is satisfied for all $t \in E_{n}$ if we take

and

$$
\alpha=\beta+m / r-\lambda \mu_{2} / r,
$$

$$
M_{2}^{r}=\int_{E_{m}}|\eta|^{\beta r}|K(|\eta|, 1)|^{\mu_{2}} d \eta .
$$

By virtue of the second relation in 3.2.1, we see easily that 3.2.2 and 3.2.4 are satisfied if and only if

$$
\lambda=n / p^{\prime}+m / r \text { and } \alpha+\left(n \mu_{2}\right) /\left(p^{\prime} r\right)=\beta+\left(m \mu_{1}\right) /\left(p^{\prime} r\right) .
$$

If we choose this value of $\lambda$, so that $K$ satisfies (i), and take

$$
\alpha=\gamma-n \mu_{2} /\left(p^{\prime} r\right), \quad \beta=\gamma-m \mu_{1} /\left(p^{\prime} r\right),
$$

where $\gamma$ is any given real number, then the hypotheses of Theorem 2.1 are satisfied with $M_{1}, M_{2}$ given by 3.2.3 and 3.2.4. We note that, by 3.2.3,

$$
\begin{aligned}
M_{1}^{p^{\prime}} & =\rho_{n} \int_{0}^{+\infty} u^{\gamma-\left(n \mu_{2} / r\right)+n-1}|K(1, u)|^{\mu_{1}} d u \\
& =\rho_{n} \int_{0}^{+\infty} u^{\gamma+\left(n \mu_{1} / p^{\prime}\right)-1}|K(1, u)|^{\mu_{1}} d u,
\end{aligned}
$$


so that $M_{1}$ satisfies (ii). Similarly $M_{2}$ satisfies (iii), and this completes the proof of the theorem.

REMARK. Since 3.2 (i) implies that

$$
|K(u, 1)|=u^{-\left(n / p^{\prime}+m / r\right)}|K(1,1 / u)|
$$

the condition 3.2 (iii) may be replaced by

$$
\rho_{m} \int_{0}^{\infty} w^{-\gamma r+\left(n \mu_{2} / p^{\prime}\right)-1}|K(1, w)|^{\mu_{2}} d w=M_{2}^{r}<+\infty .
$$

We see immediately that the integrals in (ii) and (iii) are identical if $\gamma=0$ and $\mu_{1}=\mu_{2}$. On using the second relation in 3.2.1, we obtain the following result.

THEOREM 3.3. Let $1<p \leqq r<+\infty$, let $1 / \mu=1 / p^{\prime}+1 / r$, let $m, n$ be positive integers, and let $K$ be a complex-valued function measurable on $(0, \infty) \times(0, \infty)$ such that

(i) $|K(a u, a v)|=a^{-\left(n / p^{\prime}+m / r\right)}|K(u, v)|(a>0, u>0, v>0)$,

(ii) $\int_{0}^{+\infty} v^{n \mu / p^{\circ}-1}|K(1, v)|^{\mu} d v=N<+\infty$.

Then the conclusions (i), (ii), (iii) of Theorem 2.1 hold with $S_{1}=E_{n}, S_{2}=E_{m}, \psi(x, t)=K(|x|,|t|)$, $M_{1} M_{2}=N^{1 / p^{\prime}+1 / r} \rho_{n}^{1 / p^{\prime}} \rho_{m}^{1 / r}$.

In particular, the case $r=p, m=n, \mu=1$, is Lemma 2.1 of [5].

Before considering examples to illustrate 3.3 (in the next Section), we first prove a result involving Fourier type kernels which extends Theorem 8 of [4].

THEOREM 3.4. Let $p, r, \mu_{1}, \mu_{2}$ be positive numbers such that

$$
1<p \leqq r, \quad \mu_{1} / p^{\prime}+\mu_{2} / r=1 .
$$

Let $m, n$ be positive integers, and let $L$ be a complex-valued function measurable on $(0, \infty) \times(0, \infty)$ such that

(i) $|L(u / a, a v)|=a^{m / r-n / p^{\prime}}|L(u, v)|, \quad(a>0, u>0, v>0)$,

(ii) $\rho_{n} \int_{0}^{+\infty} v^{\gamma p^{\prime}+\left(n \mu_{1} / p^{\prime}\right)-1}|L(1, v)|^{\mu_{1}} d v=M_{1}^{p^{\prime}}<+\infty$,

(iii) $\rho_{m} \int_{0}^{+\infty} u^{-\gamma r+\left(m \mu_{2} / r\right)-1}|L(u, 1)|^{\mu_{2}} d u=M_{2}^{r}<+\infty$,

where $\gamma$ is any given real number. Then the conclusions (i), (ii), (iii), of Theorem 2.1 hold with $S_{1}=E_{n}, S_{2}=E_{m}$ and $\psi(x, t)=L(|x|,|t|)$.

Proof. Suppose that, for some real number $\lambda, L$ satisfies the condition

$$
|L(u / a, a v)|=a^{-\lambda}|L(u, v)| \quad(a>0, u>0, v>0)
$$


As in 3.2, if we take $\phi_{1}(t)=|t|^{\alpha}, \phi_{2}(x)=|x|^{\beta}, \psi(x, t)=L(|x|,|t|)$, we see that condition 2.1.1 of Theorem 2.1 becomes

$$
\begin{aligned}
M_{1}^{p^{\prime}} & \geqq|x|^{-\beta p^{\prime}} \int_{E_{n}}|t|^{\alpha p^{\prime}}|L(|x|,|t|)|^{\mu_{1}} d t \\
& =|x|^{-\beta p^{\prime}-\alpha p^{\prime}-n} \int_{E_{n}}|t|^{\alpha p^{\prime}}\left|L\left(|x|,|t||x|^{-1}\right)\right|^{\mu_{1}} d t \\
& =|x|^{-\beta p^{\prime}-\alpha p^{\prime}-n+\lambda \mu_{1}} \int_{E_{n}}|t|^{\alpha p^{\prime}}|L(1,|t|)|^{\mu_{1}} d t,
\end{aligned}
$$

and this is satisfied for all $x \in E_{m}$ if we take

$$
\beta+\alpha=-n / p^{\prime}+\lambda \mu_{1} / p^{\prime}
$$

and

$$
M_{1}^{p^{\prime}}=\int_{E_{n}}|t|^{\alpha p^{\prime}}|L(1,|t|)|^{\mu_{1}} d t .
$$

Similarly, the condition 2.1 .2 is satisfied for all $t \in E_{n}$ if we take

$$
\alpha+\beta=-m / r-\lambda \mu_{2} / r
$$

and

$$
M_{2}^{r}=\int_{E_{m}}|x|^{\beta r}|L(|x|, 1)|^{\mu_{2}} d x .
$$

In view of the second relation of 3.4.1, we see that 3.4.2 and 3.4.4 are satisfied if and only if $\lambda=n / p^{\prime}-m / r$ and $\beta+m \mu_{1} /\left(p^{\prime} r\right)=-\alpha-n \mu_{2} /\left(p^{\prime} r\right)$. If we choose this value of $\lambda$, so that $K$ satisfies (i), and take

$$
\alpha=\gamma-n \mu_{2} /\left(p^{\prime} r\right), \quad \beta=-\gamma-m \mu_{1} /\left(p^{\prime} r\right),
$$

where $\gamma$ is any given real number, then the hypotheses of Theorem 2.1 are satisfied with $M_{1}$ and $M_{2}$ given by 3.4.4 and 3.4.5. Again as in 3.2, if $\rho_{n}$ denotes the surface area measure of the unit sphere in $E_{n}$, then by 3.4 .3 and the second relation of 3.4 .1 ,

$$
\begin{aligned}
M_{1}^{p^{\prime}} & =\rho_{n} \int_{0}^{+\infty} v^{\alpha p^{\prime}+n-1}|L(1, v)|^{\mu_{1}} d v \\
& =\rho_{n} \int_{0}^{+\infty} v^{\gamma p^{\prime}-n\left(1-\mu_{1} / p^{\prime}\right)+n-1}|L(1, v)|^{\mu_{1}} d v,
\end{aligned}
$$

so that $M_{1}$ satisfies (ii). We prove similarly that $M_{2}$ satisfies (iii), and this concludes the proof of the theorem.

Remark. Since 3.4 (i) implies that

$$
|L(u, 1)|=u^{n / p^{\prime}-m / r}|L(1, u)| \quad(u>0),
$$


the condition 3.4 (iii) may be replaced by

(iii)

$$
\rho_{m} \int_{0}^{+\infty} w^{-\gamma r+\left(n \mu_{2} / p^{\prime}\right)-1}|L(1, w)|^{\mu_{2}} d w=M_{2}^{r}<+\infty .
$$

We see that the integrals of 3.4 (ii) and (iii)' are identical if $\gamma=0, \mu_{1}=\mu_{2}$. On using the second relation of 3.4 .1 , we obtain the following result.

TheOREM 3.5. Let $1<p \leqq r<+\infty, 1 / \mu=1 / p^{\prime}+1 / r$, let $m, n$ be positive integers, and let $L$ be a complex-valued function measurable on $(0, \infty) \times(0, \infty)$ such that

(i) $|L(u / a, a v)|=a^{m / r-n / p^{\prime}}|L(u, v)| \quad(a>0, u>0, v>0)$,

(ii) $\int_{0}^{+\infty} v^{\left(n \mu / p^{\prime}\right)-1}|L(1, v)|^{\mu} d v=N<+\infty$.

Then the conclusions (i), (ii), (iii) of Theorem 2.1 hold with $S_{1}=E_{n}, S_{2}=E_{m}, \psi(x, t)=$ $L(|x|,|t|)$ and $M_{1} M_{2}=N^{1 / p^{\prime}+1 / r} \rho_{n}^{1 / p^{\prime}} \rho_{m}^{1 / r}$.

\section{Example.}

As stated in the introduction, we can now deduce some special cases of the other results proved in [5]. In fact we give an example which involves some of the more general results proved by Cotlar and Ortiz in [1].

It will be convenient in the definition of the fractional integral $H_{v, \theta}^{(\lambda)}(f)$ to regard the Euclidean spaces as being relatively embedded. (That is, if $m<n$, we shall regard $E_{m}$ as a subspace of $E_{n}$, so that $x \in E_{m}$ implies $x=\left(x_{1}, x_{2}, \ldots, x_{m}, 0, \ldots, 0\right) \in E_{n}$.

For $f$ in $L^{p}\left(E_{n}\right), p>1$, the integral $H_{v, \theta}^{(\lambda)}(f)$ is defined by

$$
H_{v, \theta}^{(\lambda)}(f)(x)=|x|^{(v-\theta-\lambda) n} \int_{E_{n}}|t|^{n \lambda}|t-x|^{(\theta-1) n} .(t) d t \quad\left(x \in E_{m}\right) .
$$

For $0<\theta<1$ it is easily seen that

$$
\left|H_{v, \theta}^{(\lambda)}(f)(x)\right| \leqq|x|^{(v-\theta-\lambda) n} \int_{E_{n}}|t|^{n \lambda}|| t|-| x||^{(\theta-1) n} f(t) d t .
$$

Our main result will apply to the larger kernel given by the right hand side of 4.1.1. In this case we apply Theorem 3.3 to obtain our next result.

THEOREM 4.2. Let $1<p \leqq r<+\infty, n / p^{\prime}+m / r=(1-v) n$, and suppose that the following conditions hold:

$$
0<\theta<1, \quad \lambda+\theta<1 / p<1+\lambda, \quad(1-\theta) n<1 / p^{\prime}+1 / r .
$$

Then $H_{v, \theta}^{(\lambda)}: L^{p}\left(E_{n}\right) \rightarrow L\left(E_{m}\right)$ and there is a constant $M \equiv M(\theta, \lambda, v, p, r)$ such that

$$
\left\|H_{v, \theta}^{(\lambda)}(f)\right\|_{r} \leqq M\|f\|_{p}\left(f \in L^{p}\left(E_{n}\right)\right) .
$$


Proof. In view of the remarks made earlier, this result is easily verified by taking

$$
K(u, v)=u^{(v-\theta-\lambda) n} v^{n \lambda}|u-v|^{(\theta-1) n}
$$

in Theorem 3.3. Note that in this case $-\left(n / p^{\prime}+m / r\right)=(v-1) n$ and the integral 3.3 (ii) is finite if the conditions of the theorem are satisfied.

\section{REFERENCES}

1. M. Cotlar and E. L. Ortiz, On some inequalities for potential operators, Univ. Nac. La Plata Publ. Fac. Ci. Fisicomat. Serie Segunda Rev. 8 (1962), No. 1, 16-34.

2. G. H. Hardy, J. E. Littlewood and G. Polya, Inequalities (Cambridge, 1934).

3. G. O. Okikiolu, Bounded linear transformations in $L^{p}$ space, J. London Math. Soc., 41 (1966), $407-414$.

4. G. O. Okikiolu, On certain bounded linear transformations in $L^{p^{\prime}}$, Proc. London Math. Soc. (3) 17 (1967), 700-714.

5. E. M. Stein and G. Weiss, Fractional integrals on $n$-dimensional Euclidean space, J. Math. Mech. 7 (1958), 503-514.

UNIVERSITY OF EAST ANGLIA

$\mathbf{D}$ 